\title{
UNIONES LIBRES Y MATERNIDAD OBLIGADA EN LA COSTA ECUATORIANA
}

J.Sánchez - Parga ${ }^{*}$

I ntroducimos el estudio sobre "uniones libres y maternidad obligada" con el resumen de un texto sobre la "crisis terminal de la familia en la sociedad modernd". Nos parece importante enmarcar el primer análisis en un contexto y perspectiva más generales y amplios, para mostrar en qué medida una misma institución social, como es la familia, puede encontrarse sujeta a procesos y "tiempos mixtos", y también a morfologías tan diferentes. Esto mismo nos obligaría a plantear otra cuestión: si fenómenos como las familias formadas por "uniones libres", como es el caso de la Costa ecuatoriana, constituyen residuos de una tradicional estrategia de reproducción familiar y consecuencia de una estructura agraria y organización del trabajo rural masculino del pasado, al mismo tiempo que resistencia a los procesos de destrucción de la familia en un futuro no muy lejano o si por el contrario tales modelos familiares representan más bien una de las formas que adopta, en una situación particular, la más moderna y generalizada "crisis terminal de la familia".

Desde la perspectiva de las mismas mujeres, no se debería desvincular la estrategia de las "uniones libres" con su "maternidad obligada", de ese otro fenómeno así mismo extraordinariamente complejo, por combinar también aspectos tradicionales con otros modernos: los "embarazos precoces" de las jóvenes adolescentes, que tienen lugar en ámbitos no exclusivamente costeños ni rurales, sino también urbanos y serranos y que además no se encuentran circunscritos a los sectores sociales más populares.

La hipótesis de este ensayo, y hacia donde se orienta la explicación de ambos fenómenos, no en lo que pueden relevar de más tradicional, sino en sus características y lógicas más modernas, es

\footnotetext{
* Director del Centro Andino de Acción Popular, Asesor de la Dirección Académica de la UPS Sede Quito.

1 El texto ha sido elaborado a partir de un proyecto de investigación iniciado en 1997 en el Centro de Estudios Latinoamericanos de la PUCE sobre "Poligamia y fecundidad en el Ecuador" y que no fue concluido.
} 
que tanto las "maternidades obligadas" de las "uniones libres" como los "embarazos precoces" de las adolescentes, responden hoy a una estrategia de integración de la mujer joven en la sociedad.

A diferencia de los varones, cuyo "síndrome adolescente" para forzar su integración a la sociedad (y para forzar también formas más o menos reales y simbólicas de independencia respecto de la familia) adquieren manifestaciones extremadamente violentes y de morfologías de violencia muy diversas, la mujer adolescente habría intentado una modalidad de integración, no muy diferente a las formas más tradicionales, también forzada pero con un género de violencia muy diferente al de los jóvenes: menos trasgresor, delincuencial o suicidiario, pero no por ello menos grave y de consecuencias igualmente dolorosas ${ }^{2}$.

Frente a las dificultades de integración en la sociedad, la mujer adolescente encontraría en la maternidad precoz una estrategia para independizarse de su familia o lograr una cierta autonomía aun dentro de ella, con una también relativa "incorporación" a la sociedad, en la medida que participa en el ciclo de su reproducción.

Un dato antropológico muy generalizado en las culturas más diversas destaca la importancia del síndrome maternal y de maternidad en la socialización de la mujer y en su integración a las sociedades tradicionales. En este sentido resulta muy singular que en la sociedad moderna las adolescentes fuercen su integración a ella, recurriendo a una estrategia tradicional, la cual en muchos aspectos se muestra tan contradictoria como disfuncional respecto de las características de la sociedad actual. De ahí que tanto la "maternidad obligada" de las "uniones libres" como los "embarazos precoces" tengan que ser pensados, comprendidos y explicados, no tanto desde las jóvenes adolescentes que los provocan, cuan-

\footnotetext{
2 En dos textos anteriores nos hemos ocupado respectivamente de la violencia adolescente de los jóvenes (cfr. J. J. Sánchez - Parga, "Identidades y violencias adolescentes" en C. Landázuri, Memorias del Primer Congreso Ecuatoriano de Antropología, PUCE I MARKA, Abya - Yala, Quito, 1998), y de la problemática adolescente en cuanto problema de su integración por parte de la sociedad moderna (Transferencia antropológica y síndrome adolescente de la sociedad, UPS, Quito, próxima publicación).
} 
to desde la sociedad que los propicia, los produce, los generaliza, los vuelve necesarios (?), ante otras alternativas posibles de integración adolescente.

Así establecido el marco explicativo más general, prescindiremos por el momento del caso relativo al "embarazo precoz", para ocuparnos de esa "maternidad obligada" y sus "uniones libres". Aunque también este mismo fenómeno reviste problemáticas complejidades en la medida que involucra de una u otra manera todas las relaciones familiares (madre, el progenitor, el padre y los hijos, en su doble condición femenina y masculina).

\section{Una crisis terminal de la familia}

La peculiar característica de la crisis actual de la familia contradice las tesis clásicas y conservadoras, según las cuales es la crisis de valores, dilema moral, la principal causa del conflicto familiar. Datos y estadísticas en Ecuador, Europa y EEUU, como en casi todo el mundo, demuestran que son las crisis por las que atraviesan las relaciones familiares y las relaciones de la familia con la misma sociedad, el problema del modelo de socialización al interior de la misma familia, lo que genera una crisis ética y de valores, y no al contrario. Por otro lado, lo que está realmente en aprieto, y tal es la novedad actual, no es uno u otro modelo de familia, sino la misma institución familiar. El carácter estructural de dicha crisis hace que sean los elementos constitutivos de la familia, la específica naturaleza de las diferentes relaciones entre sus miembros (alianza del contrato matrimonial, la filiación y paternidad, y la consanguinidad entre hermanos), lo que representa una real mutación de la familia en la sociedad moderna.

La crisis de la familia se encuentra atravesada por varios ciclos, que lejos de sucederse en el tiempo, se superponen y se acumulan y condensan. Un primer ciclo crítico, de largo plazo, supuso el paso de la familia extensa o ampliada a la familia nuclear, mientras que el más reciente supone una creciente marginación de la misma familia nuclear y su contracción tanto en su volumen o número de sus miembros como en el tiempo de su duración. La novedad de estas más recientes transformaciones ha sido conceptuali- 
zada como "desinstitucionalización" o "tiempos de la incertidumbre" para la familia moderna ${ }^{3}$.

Mientras que la crisis de larga duración redujo las funciones de la familia, la crisis más reciente y también más acelerada no solo reduce el número y tamaño de la unidad doméstica, sino que también tiende a modificar el mismo orden y representación psico-sociológica de la familia, al eliminar de ella uno o varios de sus integrantes fundamentales: el padre, la madre o el/la hijo/a y los hermanos.

La secular dinámica de "desfamiliarización de la sociedad" comienza a acelerarse desde mediados del siglo XIX. La separación entre el trabajo y el hogar, iniciada con la aparición de la fábrica, arrebata a la familia su función productiva, y la aparición del salario reduce la importancia del patrimonio familiar, debilitando su economía. Los procesos de movilidad social (vertical y horizontal) y geográfica rompen los vínculos de la familia extensa, separando padres, hijos/as y hermanos/as; y en fin los procesos de socialización primaria y secundaria que monopolizaba la familia, garantizando la integración de los hijos/as al espacio público de la sociedad, han quedado en gran medida transferidos a la escuela, a los medios de comunicación y a los grupos etarios. Estos son los que cada vez de manera más exclusiva socializan a los/as niños/as y adolescentes, se convierten en mediaciones de su integración a la sociedad, despojando a la familia y a los padres de esta su principal función y competencia.

La familia parece quedar hoy reducida a una unidad mínima de consumo (ya no de producción) y a la crianza de los/as menores (y cada vez menos a su socialización).

El ciclo más reciente de la crisis, mucho más importante y resultado de tres factores biológicos y de tipo médico sanitario, incide en el número y tamaño de la familia, y por consiguiente en la masa de cohesión familiar y en su peso específico al interior de la sociedad.

En primer lugar, el alargamiento de la esperanza de vida duplicado en el último siglo (pasando en los países desarrollados de los

\footnotetext{
3 Para un amplio desarrollo y análisis más detallado de lo que se plantea a continuación, puede consultarse el gran clásico sobre el tema, con su original enfoque demográfico: Louis Roussel, La famille incertaine, Edit. Odile Jacob, Paris, 1989.
} 
35 a los 78 años) significa que la reproducción sistémica de la sociedad se reduce a menos de la mitad: antes una sociedad o grupo humano debía reproducirse cada 36 años, mientras que ahora lo hace cada 78 años. Según esto, si la familia constituye la unidad básica de la reproducción social, hoy se necesitan menos de la mitad de familias que hace un siglo. En consecuencia, menos familias y menos hijos por familia.

A esto contribuye, en segundo lugar, el descenso de la mortalidad infantil. Hace 90 años se requerían entre 4 y 5 hijos para que 2 de ellos llegaran a la edad de reproducción; hoy solo se necesitan 2,1 hijos para cumplir dicho ciclo reproductivo. Esto, unido a los métodos de control de la natalidad, hace que el tiempo dedicado a la reproducción se reduzca muy considerablemente, y por consiguiente la actividad social de la familia se independice de su actividad reproductiva.

Otra importante consecuencia para la liberación de la mujer, es que antes tenía que dedicar la totalidad de su vida adulta a la procreación y crianza de los hijos, pues no vivían más de 40 años; hoy basta a las mujeres tener 2,1 hijos para garantizar la reproducción y además viven más de 70 años. Esto explica que la incorporación de las mujeres al estudio, la profesionalización y al trabajo no se haya realizado a costa del abandono de la vida familiar sino disponiendo de un tiempo excedente.

Este reciclamiento biológico en el modelo de reproducción tiene otras consecuencias, al afectar la naturaleza misma del vínculo matrimonial. Mientras que las parejas antiguas solo podían esperar 15 ó 20 años de vida en común, las actuales cuentan con una esperanza de convivencia de 30 a 40 años promedio. A esto hay que añadir un factor adicional: mientras que antes toda la vida en común de la pareja estaba muy vinculada a la presencia de los hijos en el hogar, en la actualidad la prolongación de la esperanza de vida hace que gran parte del ciclo matrimonial transcurra sin la presencia de los hijos.

En Europa el 25\% de hogares son unipersonales, y más del 33\% en los países nórdicos, donde además el $50 \%$ de los/as niños/as son hijos de madres solteras; en París, declarada, como Manhattan, "la capital de la soledad", el $50 \%$ de hogares son unipersonales. Todos estos fenómenos que caracterizan un nuevo modelo de 
"sociedad desfamiliarizada", donde la familia como institución privada pierde peso e importancia públicos, dan lugar a un modelo de sociedad crecientemente hiper-individualizado, donde el peso e importancia se centra en los individuos particulares, autónomos y aislados. Son estos fenómenos los que preparan e introducen el ciclo terminal de la crisis familiar en la sociedad moderna.

Estos cambios tienen consecuencias socio-culturales no fáciles de prever. Siendo la familia la única institución que no funciona según el principio de reciprocidad, donde lo que se dona no está sujeto a retribución ni devolución, la reducción y el declinar de la familia en parte es efecto, pero en parte es también factor de la creciente mercantilización del orden social. La moderna sociedad de mercado es incompatible con la sociedad familiar.

No se puede hablar de una crisis de la familia que no corresponda a una crisis de sociedad, puesto que aquella no solo refleja esta sino también el modelo de articulación e integración entre la sociedad y la familia. Es sobre todo este último aspecto el que hoy adquiere efectos más determinantes, ya que afectan la disfuncionalidad de la familia dentro de la sociedad moderna, donde aquella tiende a convertirse en un cuerpo o institución extraño dentro de esta. Lo que hoy se alteran son las específicas relaciones de parentesco constitutivas de la familia: son las relaciones conyugales o alianza matrimonial, de filiación y paternidad, de consanguinidad o fraternas, las que o bien desaparecen o están siendo profundamente alteradas. Las "uniones" o las parejas han dejado de ser conyugales y matrimoniales, y las mismas alianzas han perdido contractualidad para volverse "compromisos". Las ilimitadas posibilidades de la manipulación genética han mutado las nociones de paternidad y de progenitura y hasta de maternidad ${ }^{4}$.

La moderna sociedad de mercado, la "sociedad en redes" (network society según Castel) tiende a desvincular todo tipo de "lazo social", y toda corporación, toda pertenencia y contractualidad, toda relación y organización, para producir individuos autónomos frente a la única o dominante organización social del mercado, donde todo se compra y todo se vende, donde todo es oferta y demanda. Frente a este mo-

\footnotetext{
4 Hoy una mujer sola puede concebir un hijo sin necesidad de conocer al progenitor, en algunos países parejas de homosexuales de ambos sexos "casados" o en unión libre pueden convertirse en padres legales de hijos no concebidos por ninguno de ellos.
} 
delo de sociedad la familia o desaparece o se pervierte, ya que frente al mercado todo individuo o ciudadano pierde todas sus otras condiciones, plenamente no puede ser más que un productor y consumidor.

Es en el contexto global de estos procesos transformadores del modelo familiar, que se vuelve tan contrastante como singular el caso de una formación familiar basada en el modelo de las "uniones libres", tal y como se encuentra en las provincias costeras del Ecuador.

\section{Las "uniones libres" y la "maternidad obligada"}

Dentro del elevado porcentaje de "uniones libres" en el país, $14 \%$ según el Censo de 1990 entre la población femenina mayor de 12 años, llama la atención que este "estado civil" responda menos a un fenómeno de modernización de la sociedad y la familia, que a una razón y tradición culturales, ya que aparecen sobre todo como una situación muy regionalmente circunscrita, las provincias de la Costa, y predominantemente rural $(16,30 \%)$ y no tanto urbana $(11,7 \%)$.

\section{Uniones Libres. Distribución Regional y por Áreas: 1990}

\begin{tabular}{|c|l|c|}
\hline REGIONES & ÁREAS & \multicolumn{1}{c|}{$\%$} \\
\cline { 2 - 3 } Sierra & Total & $4,0 \%$ \\
& Urbano & $4,0 \%$ \\
& Rural & $4,0 \%$ \\
\hline \multirow{2}{*}{ Costa } & Total & $26,0 \%$ \\
& Urbano & $21,5 \%$ \\
\hline \multirow{3}{*}{ Oriente } & Rural & $30,4 \%$ \\
& & \\
& Total & $9,1 \%$ \\
& Urbano & $7,6 \%$ \\
& Rural & $10,0 \%$ \\
\hline
\end{tabular}

Fuente: Censo 1990

En apoyo a la hipótesis sobre el carácter tradicional de este fenómeno tres géneros de informaciones cabe aducir:

a) Una distribución muy diferenciada del fenómeno en las tres grandes regiones del país: mientras que en las provincias de la Sierra el promedio de uniones libres es del 3,6\%, en las provincias de la Costa es del $26,9 \%$, y en el Oriente es del $12,6 \%$. 
b) Los datos censales de 1950 y 1962 arrojan un porcentaje todavía mayor de uniones libres, en la Costa, $28,0 \%$ y $25,2 \%$ respectivamente, que los registrados en el Censo de 1990; y el hecho ya mencionado trata de un fenómeno predominantemente rural y no urbano.

c) El hecho de que el porcentaje de divorcios sea relativamente bajo en el país $(0,8 \%)$ y obviamente inferior en las áreas rurales costeñas $(0,3 \%)$.

Se trata de una situación generalizada en las cuatro provincias de la Costa, donde las uniones libres representan el $41 \%$ en Esmeraldas, el $28,3 \%$ en Guayas, el $40,8 \%$ en Los Ríos y el $27,4 \%$ en Manabí. En el Oriente los porcentajes se distribuyen de la siguiente manera: Napo 9,6\%, Pastaza, 9,9\%, Sucumbíos, 18,3\%.

Las "uniones libres" en las provincias de la Costa y muy particularmente en las áreas rurales no son tan libres como pudieran parecer, puesto que se encuentran muy condicionadas al nacimiento de un/a hijo/a. De hecho una unión marital nunca genera un "compromiso" si de ella no nace un/a hijo/a. Esto demuestra que es sobre todo el/la hijo/a, el que actúa como vínculo en las uniones libres, y lo que genera el real compromiso del progenitor respecto del/la hijo/a tanto como con la madre ${ }^{5}$. Esta doble vinculación explica que, disuelta una unión libre por las razones que fuere, la mujer/madre solo establece otra nueva unión en la medida que un/a nuevo/a hijo/a nace de una nueva relación con otro hombre.

Las "uniones libres" se organizan de acuerdo a una estructura etaria femenina muy particular, que va de los 15 a los 19 , con un promedio del $44,4 \%$ de la población, mientras que entre los 45 y 49 años de edad el porcentaje de uniones libres respecto de los otros "estados civiles" es del 22,4\%.

\begin{tabular}{|c|c|c|c|c|c|c|}
\hline & \multicolumn{4}{|c|}{ Grupos de Edad: } & \multirow[b]{2}{*}{$\begin{array}{c}35-39 \\
\text { años }\end{array}$} & \multirow[b]{2}{*}{$\begin{array}{c}39-49 \\
\text { años }\end{array}$} \\
\hline & $\begin{array}{c}15-19 \\
\text { años }\end{array}$ & $\begin{array}{c}20-24 \\
\text { años }\end{array}$ & $\begin{array}{c}25-29 \\
\text { años }\end{array}$ & $\begin{array}{c}30-34 \\
\text { años }\end{array}$ & & \\
\hline $\begin{array}{l}\text { Uniones } \\
\text { Libres }\end{array}$ & $44,4 \%$ & $34,3 \%$ & $29,1 \%$ & $26,4 \%$ & $25,7 \%$ & $22,4 \%$ \\
\hline
\end{tabular}

Fuente: Censo 1990

5 Hemos desarrollado más ampliamente la función simbólica del hijo como vínculo matrimonial en un estudio anterior: "El vínculo conyugal en las actuales sociedades andinas", en La letra. Revista de psicoanálisis, n. 1, Quito, 1995. 
Esto significa, según la más espontánea de las hipótesis, que en el transcurso de sucesivas uniones libres, con sus respectivos "compromisos" (e hijo/as) se van estableciendo nuevas uniones matrimoniales, por lo general con esposos mucho mayores, que ya han tenido anteriores uniones libres y que frecuentemente son viudos, están o estuvieron casados. Sin embargo la hipótesis más verosímil y apoyada por estimaciones censales obligaría más bien a supone, que, concluida una "unión" y no sustituida por otra, las mujeres se declaran solteras manteniendo su condición de madres. Es muy improbable que no solo en la Costa sino también en las áreas rurales de la Sierra aumente el número de madres solteras después de los 19 años.

La precocidad de las uniones libres en la situación estudiada comporta una maternidad prematura y obviamente también elevadas tasas de natalidad, si se tiene en cuenta que el "vínculo" o "compromiso" que establece la unión libre se establece, se refuerza y se perpetúa en la medida que perduran las responsabilidades de los progenitores respecto de lo-as hijo-as nacidos de tales uniones. En este sentido, no solo la maternidad prematura, a partir de los 15 años, propicia elevadas tasas de natalidad, sino también la necesidad de reanudar la unión libre con nuevos hijo/as o de reiniciar otra nueva unión libre con otro hombre, que obliga a un nuevo nacimiento.

\section{REGIÓN COSTA: POBLACIÓN FEMENINA DE 15 AÑOS 0 MÁS, POR ESTADO CIVIL O CONYUGAL, POR NÚMERO DE HIJOS NACIDOS VIVOS, SEGÚN ÁREA}

\begin{tabular}{|l|r|r|r|r|}
\hline \multirow{2}{*}{$\begin{array}{l}\text { ESTADO CIVIL } \\
\text { O CONYUGAL }\end{array}$} & \multicolumn{2}{|c|}{ URBANO } & \multicolumn{2}{c|}{ RURAL } \\
\cline { 2 - 5 } & VALOR & \multicolumn{1}{c|}{$\%$} & \multicolumn{1}{c|}{ VALOR } & \multicolumn{1}{c|}{$\%$} \\
\hline UNIDA & 215481 & $21,92 \%$ & 185892 & $38,20 \%$ \\
\hline SOLTERA & 313456 & $31,89 \%$ & 120289 & $24,72 \%$ \\
\hline CASADA & 335102 & $34,09 \%$ & 134127 & $27,57 \%$ \\
\hline DIVORCIADA & 14360 & $1,46 \%$ & 1.82 & $0,33 \%$ \\
\hline VIUDA & 48244 & $4,91 \%$ & 22186 & $4,56 \%$ \\
\hline SEPARADA & 38532 & $3,92 \%$ & 13267 & $2,73 \%$ \\
\hline NO DECLARADO & 17902 & $1,82 \%$ & 9232 & $1,90 \%$ \\
\hline TOTAL & $\mathbf{9 8 3 . 0 7 7}$ & $\mathbf{1 0 0 , 0 0} \%$ & $\mathbf{4 8 6 . 5 7 5}$ & $\mathbf{1 0 0 , 0 0 \%}$ \\
\hline
\end{tabular}

Fuente: INEC, Censo de Población, 1990.

Elaboración: Belisario Torres. -CAAP- 
REGIÓN COSTA: NÚMERO DE HIJOS NACIDOS VIVOS SEGÚN ESTADO CIVIL O CONYUGAL DE LA MADRE, POR ÁREA.

\begin{tabular}{|l|r|r|r|r|}
\hline \multirow{2}{*}{$\begin{array}{l}\text { ESTADO CIVIL } \\
\text { O CONYUGAL }\end{array}$} & \multicolumn{2}{|c|}{ URBANO } & \multicolumn{2}{c|}{ RURAL } \\
\cline { 2 - 5 } & \multicolumn{1}{c|}{ VALOR } & \multicolumn{1}{c|}{$\%$} & \multicolumn{1}{c|}{ VALOR } & \multicolumn{1}{c|}{$\%$} \\
\hline UNIDA & 742949 & $29,38 \%$ & 852876 & $47,29 \%$ \\
\hline SOLTERA & 157877 & $6,24 \%$ & 49548 & $2,75 \%$ \\
\hline CASADA & 115852 & $45,71 \%$ & 671250 & $37,22 \%$ \\
\hline DIVORCIADA & 42555 & $1,68 \%$ & 6510 & $0,36 \%$ \\
\hline VIUDA & 266562 & $10,54 \%$ & 154288 & $8,55 \%$ \\
\hline SEPARADA & 141056 & $5,58 \%$ & 54602 & $3,03 \%$ \\
\hline NO DECLARADO & 21938 & $0,87 \%$ & 14431 & $0,80 \%$ \\
\hline TOTAL & $\mathbf{2 5 2 8 7 8 9}$ & $\mathbf{1 0 0 , 0 0 \%}$ & $\mathbf{1 8 0 3 5 0 5}$ & $\mathbf{1 0 0 , 0 0 \%}$ \\
\hline
\end{tabular}

Fuente: INEC, Censo de Población, 1990.

Elaboración: Belisario Torres. -CAAP-

Uno de los problemas que reproduce la "unión libre", y que opera dentro de un círculo vicioso, son las tasas elevadas de deserción escolar y los bajos niveles de instrucción de las mujeres: aun cuando el sector femenino se ha incorporado masivamente a la instrucción escolar en las últimas décadas, sin embargo siguen siendo bajos los niveles de instrucción que alcanzan las mujeres, ya que abandonan el colegio con el primer embarazo.

Mientras que en el sector urbano de las provincias de la Costa el número de madres casadas $(34,0 \%)$ es muy superior al de las solteras $(31,89 \%)$ y de las unidas $(21,92 \%)$, en el sector rural se invierten significativamente los porcentajes: mucho mayor el de madres unidas $(38,2 \%)$ que el de casadas $(27,57 \%)$, mientras que el de madres solteras representa el $24,7 \%$. Cifra esta última demasiado elevada y que solo se entiende dentro de una biografía o secuencia de "uniones libres".

Por lo que respecta a la distribución de la natalidad en los diferentes estados civiles, los datos sobre el número de hijos/as (nacidos/as vivos/as) guarda una estrecha correspondencia con los del cuadro anterior: en el sector rural el $47,29 \%$ de los hijos pertene- 
cen a "uniones libres", el $37,22 \%$ a mujeres casadas, el $2,75 \%$ a solteras. Lo que supone una de natalidad y conyugado contrarios a los del sector rural, donde el $45,71 \%$ de los hijos son de madres casadas, el $29,38 \%$ de madres unidas, el $6,24 \%$ de solteras.

Si finalmente consideramos el promedio de hijos de acuerdo al estado civil de la madre, encontramos que en el sector urbano el número de hijos es el mismo para las madres "unidas" que para las madres "casadas": 3,4; mientras que en el área rural, donde el número de hijos es muy superior, el promedio para las madres unidas es del 4,6 y para las casadas de 5,0.

Otro fenómeno singular es que las mujeres sin instrucción escolar tienden a establecer la primera unión libre con la consiguiente maternidad cuatro o cinco años antes que las mujeres con más de 7 años de instrucción escolar. Con una particularidad respecto a estos datos del Censo de 1979: que en la actualidad la primera unión libre y maternidad tiende a anticiparse aún más, pasando del promedio de los 19 años de edad al de 15 años de edad, lo que, de acuerdo a informaciones preliminares, respondería a una mayor movilidad femenina, para la que el mismo colegio proporciona mayores ocasiones de libertad a las jóvenes.

También de acuerdo a los Censos de 1979 / 80 las mujeres sin actividad económica tienden a contraer la primera unión libre a una edad inferior a los 18 años, mientras que las mujeres con cualquier tipo de ocupación laboral contraen su primera unión libre después de los 21 años de edad.

\section{Algunas pistas interpretativas}

Del fenómeno analizado se han propuesto dos tipos de explicaciones. Una de carácter estructuralista, que parece inspirada en el modelo propio de sociedades más tradicionales, argumenta que en una socio-cultura donde la mujer solo se incorpora plenamente a la sociedad en cuanto madre, tanto más si las mismas condiciones socio-económicas y culturales limitan su acceso a la educación, a la profesionalización y los desempeños laborales, su única estrategia de vida consiste en fortalecer su posición en la familia y dominar el ámbito del hogar y la economía doméstica, establecien- 
do un vínculo con el hombre y con la sociedad a través del/la hijo/a o los/as hijos/as que tenga de él. De esta manera, las "uniones libres" de una "maternidad obligada" permiten la subsistencia de la mujer y su realización como madre. Esto mismo justificaría que el hombre mantenga varias uniones libres y todo un sistema de "compromisos" y "obligaciones" con los/as hijos y sus madres, que garantizan la reproducción monoparental de diferentes hogares.

Tal explicación muy bien puede justificarse y completarse en base de las características socio-económicas y culturales de las provincias de la Costa, su estructura agraria y sistemas agrícolas y de cultivos, que requieren una gran movilidad espacial del hombre, y donde las condiciones laborales de la mujer fuera del hogar son muy limitadas. Solo en décadas actuales una relativa industrialización del sector agrario junto con la ampliación de los transportes y niveles relativamente superiores de la instrucción femenina parecen haber ampliado las posibilidades laborales del sector femenino.

Otra interpretación del mismo fenómeno, que más que excluir complementa la anterior, se refiere al carácter trashumante de la mano de obra masculina, obligada a constantes y más o menos largos desplazamientos a causa de las zafras, estaciones de cosecha, recolección diferente y distantes zonas de la misma región, lo cual propiciaría el establecimiento de "uniones libres" y hogares en algunos de los sitios por donde suele desplazarse con mayor regularidad la mano de obra masculina e instalarse temporalmente a causa del trabajo. Tal hipótesis se encontraría confirmada por el hecho de que tal fenómeno tiene también lugar en regiones con características similares en los países centroamericanos y del Caribe.

Sin embargo, esta explicación no parece por si sola suficiente, sobre todo debido a la facilidad de los transportes en épocas recientes. Además porque el fenómeno de las "uniones libres" tiene así mismo lugar en zonas donde la movilidad laboral no es tan frecuente y, también aunque en menor proporción en regiones con distintas características de trabajo. Es posible y verosímil que un tal seminomadismo de los hombres en épocas anteriores haya 
contribuido a fraguar modelos de matrimonios y de "uniones libres", que actualmente responden a otras razones y motivaciones, tanto por parte de los hombres como de las mujeres.

Sin descartar la general o parcial relevancia explicativa de estas interpretaciones, no hay que dejar de referirse a las identidades familiares que dicho fenómeno produce. Ya que la "unión libre" no solo se encuentra profundamente arraigada en la cultura costeña, sino que además goza de una particular valoración ideológica entre las mismas mujeres, para las cuales el matrimonio representa una relación que además de mantenerla en una desventajosa dependencia respecto del marido, supone una permanente inseguridad y alto riesgo de abandono, condición esta que la mujer vive de manera humillante. En la "unión libre" la mujer goza de una relativa (simbólica o imaginaria) condición de libertad respecto de su compañero, que no tendría en su condición de casada. Pero más importante todavía es que la "unión libre" le proporciona la posibilidad de entablar nuevas y sucesivas relaciones libres con nuevos compañeros, capitalizando nuevos "compromisos" y "obligaciones" por parte de ellos, lo que no parece proporcionar la condición de esposa abandonada o divorciada.

La "unión libre" significa para la joven mujer o niña la única ocasión y estrategia de independizarse de su familia, y en tal sentido comporta un estado de autonomía y de "mayoría de edad", casi ritual de pasaje, que de otra manera nunca lograría.

Dos razones complementarias contribuyen a enriquecer y corroborar estas explicaciones. Por un lado, la ausencia de una dimensión contractual dentro del vínculo matrimonial, representado más bien como un "compromiso" a partir de la existencia de un/a hijo/a, la condición de matrimonio se representa tan débil como poco significativa, mientras que se refuerza la condición de madre y la de progenitor, haciendo que la identidad femenina se realice y se consuma en la maternidad, mientras que la identidad masculina se realiza y consuma no tanto en la paternidad, en cuanto cualificación de la relación con los/as hijos/as, cuanto en la progenitura cuantitativa, referida más bien al mayor número de hijos/as (asociado imaginariamente con el mayor número de mujeres). 
La fuerte identidad materna de la mujer hace que se refuerce en el hombre una correspondiente identidad de progenitor. Solo la masculinidad progenitora en parte complementa y en parte justifica la feminidad materna. De esta manera ambas identidades de género se construyen en referencia a una particular alteridad: es en referencia recíproca de la mujer / madre y del varón / progenitor que la situación de las uniones libres de maternidad obligada pone de relieve la constitución de las identidades de género en la familia de las sociedades rurales de la Costa.

Hay que reconocer que la fuerte e intensa identificación materna, que puede volverse tan exclusiva de la identidad femenina no es un fenómeno restringido a las regiones de la Costa, ni tampoco asociado a las "uniones libres", sino que se encuentra también muy arraigado en sectores femeninos de la Sierra tanto indígenas como mestizos. Mujeres de sectores populares suburbanos de Cuenca reaccionaron violentamente contra un programa de planificación de la natalidad con un grito muy significativo: "nos quieren quitar lo único que podemos hacer, lo mejor que sabemos hacer": los/as hijos/as. Mientras que en otros sectores vecinos, donde los niveles de instrucción y de las actividades laborales y profesionales de las mujeres se habían ampliado muy sensiblemente, dicho programa de planificación contó con un relativo éxito, ya que en este sector las identidades femeninas poseían además de la maternidad otros referentes de identificación.

Tradicionalmente la unión libre se establecía a partir de la primera relación sexual, que en el mejor de los casos comportaba el primer embarazo, con el agravante de que una primera relación sexual sin embarazo se consideraba una desgracia que podía perjudicar el futuro de la joven adolescente ${ }^{6}$. Tal comportamiento tiende en la actualidad a modificarse, porque una mayor movilidad femenina y libertad sexual, debida en gran parte a la escolarización en la instrucción media, proporciona a la joven relaciones sexuales más precoces, que se combina con una prostitución adolescente. Se ha vuelto muy usual que a la salida de los colegios las jóvenes se prostituyan ocasionalmente (algunos "lances" semanales) con la única y simple finalidad de obtener dinero fácil para "sus compras".

\footnotetext{
6 Nada ilustra mejor el dramatismo de esta situación, por muy cruel que parezca, que el dicho costeño "desgraciada la mujer culeada, pero no embarazada".
} 
La "unión libre" se encuentra sujeta a una coacción más o menos compulsiva por parte de la propia familia de las jóvenes, con la finalidad de aliviar en muchos casos la carga doméstica del hogar. Esta situación introduce un dilema dentro de la misma estrategia de la "unión libre", que obliga a conjugar el criterio de urgencia con el criterio de selección.

Al "excedente de maternidad" que se condensa en torno a las "uniones libres" corresponde, un "déficit de paternidad", lo que plantea una particular problemática no sólo de índole socio - cultural, por lo que representa el padre en los procesos de socialización de los hijos, sino también familiar, por lo que se refiere a la coherente o completa formación y funcionamiento de la familia ${ }^{7}$.

La ausencia del padre supone la supeditación de las relaciones de paternidad por las del progenitor, y la débil presencia o regular ausencia de este en el hogar, su inestabilidad o temporalidad.

Hay que tener en cuenta que en no pocas ocasiones el mismo progenitor mantiene dos o más "uniones libres" simultáneamente en hogares diferentes. Mientras que por parte del hombre varias uniones libres pueden mantenerse simultáneas, en el caso de la mujer solo sucesiva o secuencialmente se establecen las "uniones libres" con hombres diferentes. En ambos casos, y de acuerdo a estimaciones provisionales, las uniones libres tienden a estabilizarse con la edad de uno o de ambos miembros de la pareja.

Sin aventurarnos a establecer una asociación demasiado estrecha y directa entre las "uniones libres" y el incesto, ya que esta problemática transciende aquel fenómeno y rebasa incluso sus condiciones socio regionales, hay datos suficientes para considerar que la frecuencia del incesto, por no decir su amplia generalización en la región costeña y sus áreas rurales, se encuentra muy determinado por la situación familiar que configuran las "uniones libres".

\footnotetext{
7 Si entendemos la familia, no como "un grupo de personas que vive bajo un mismo techo y bajo una misma autoridad", según la definición del Diccionario de la Academia de la Lengua, sino como una agrupación de personas que se relacionan por vínculos de alianza, de filiación y consanguinidad.

8 Es una opinión muy generalizada en la provincia de Manabi que la primera relación sexual de toda niña es con su padre o padrastro, relación que queda sometida a la más estricta ley del silencio, por la supuesta pero perversa consideración de que "lo que ocurre en familia es siempre menos grave".
} 
La presencia de progenitores y padrastros en la familia hace que la figura del incesto pueda atribuirse tanto a unos como a otros. Con el agravante de que en no pocos casos dicho incesto entre progenitor o padrastro y su hija cuenta con la complicidad más o menos tácita de la madre, que la entrega o abandona sexualmente al padre, ya sea resignada o interesada, pero con la finalidad de seguir manteniendo a su compañero en la casa. Es la ausencia de una paternidad simbólicamente vivida la que hace posible y despoja de su perversión el incesto o violación entre el progenitor y su hija.

El problema del incesto al interior de las familias formadas por "uniones libres" presenta una doble fisonomía: si por una parte parece mucho más traumático, cuando tiene lugar antes de la pubertad de la hija, ofrece otra gravedad cuando dicho incesto da lugar al nacimiento de un hijo que es a la vez hermano de la madre y nieto del progenitor. Esta confusión de todas las relaciones familiares, es lo que genera problemas o traumas más profundos de identidad.

Aunque no haya cifras más que estimativas, dada la dificultad de producir tal tipo de información, por encuestas realizadas se calcula que una de cuatro niñas menores de 12 - 14 años en las áreas rurales costeñas, han sido violadas por el padre, y dos de cada cinco después de los 14 años. Hasta tal punto que es opinión generalizada en las áreas rurales que el incesto representa una suerte de derecho paterno y de rito obligado e inicial de las hijas ${ }^{9}$.

Estos resultados de un avance de investigación tendrían que ser completados con nuevas informaciones y más datos, sobre todo relativos a cómo los diferentes sectores de una sociedad y los distintos miembros de la familia perciben y viven estos fenómenos tan complejos y de consecuencias tan diversas para la existencia de

\footnotetext{
9 Que la práctica del incesto se extiende más allá de las regiones costeñas y sus áreas rurales, atravesando toda clase social, a excepción de los sectores indígenas, es algo que se puede inferir del trabajo clínico del psicoanálisis, y de un síndrome muy generalizado que hemos Ilamado "un intruso en el tálamo" (cfr. La familia en las sociedades andinas: sus crisis mixtas, PUCE / CELA, Quito, 1997). Sobre el sigilo social que rodea la cuestión del incesto puede consultarse Álvaro Carrión, "Silencio" en Psicoanálisis y Sociedad, Ecuador Debate, n. 52, Abril, 2001.
} 
todos ellos. Dicho fenómeno debería incluso ser a su vez analizado en un contexto socio-cultural más amplio y comparativamente relacionado con otras morfologías similares de la región latinoamericana ${ }^{10}$.

Finalmente, resultados de una investigación reciente en sectores de comunidades indígenas de la Sierra ecuatoriana han puesto de manifiesto un fenómeno relativamente nuevo en sus formas y frecuencias dentro de la tradicional cultura andina, y que aparece como consecuencia de modernas transformaciones de la sociedad comunal con su efecto desestructurador de las relaciones familiares, y de manera muy particular del mismo vínculo matrimonial ${ }^{11}$.

Se trata del creciente número de jóvenes madres, que no terminan casándose (como es habitual en el medio indígena, donde el/la hijo/a es, por lo general, condición o precursor del matrimonio) o que después de casadas, pero en un tiempo relativamente corto, son abandonadas. Sin atrevernos a asimilar esta fenomenología indígena a la descrita en la región de la Costa, no cabe ignorar ciertas analogías, aun cuando se trata de procesos diferentes. Pero lo que resulta evidente tanto en términos sociológicos como antropológicos, en todos los casos, es la estrecha simetría de asociaciones $u$ oposiciones entre maternidad y matrimonios.

\footnotetext{
10 En la sociedad cartagenera de Colombia, por ejemplo, el hecho de mantener una "unión libre", pero no necesariamente con hijo, por parte del varón casado se encuentra tan profundamente arraigado y legitimado, que para una esposa sería casi inconcebible y hasta socialmente embarazoso, que su marido no mantuviera una relación extramatrimonial con otra mujer. Como si fuera parte de un "prestigio" del marido, que la propia esposa no puede dejar de compartir.

11 Cfr. J. Sánchez - Parga, Crisis en torno al Quilotoa: mujer, cultura y comunidad, CAAP, Quito, 2002.
} 
